\title{
一种溶酶体靶向双光子亚硝酰氢荧光探针的合成及细胞成像研究
}

\author{
王晓芬魏超* 李雪艳郑雪阳耿晓维 \\ 张平竹李小六*
}

(河北大学化学与环境科学学院 药物化学与分子诊断教育部重点实验室 河北省化学生物学重点实验室

保定 071002)

\begin{abstract}
摘要 亚硝酰氢( $\mathrm{HNO}$ )是一氧化氮单电子还原并质子化的产物, 具有重要的生物学意义. 以 4-(2-氨基乙基)-吗啉作为 溶酶体靶向基团, 1,8-菜二甲酰亚胺作为双光子荧光团, 三苯基膦作为 HNO 识别基团, 构建了一个能够特异性定位于 溶酶体的打开型双光子荧光探针 Lyso-HNO. 研究结果表明, 该探针响应迅速, 对 HNO 表现出良好的选择性, 较高的 灵敏性, 检测极限可达 $202 \mathrm{nmol} \cdot \mathrm{L}^{-1}$. 该探针可对 HeLa 细胞溶酶体外源 HNO 进行双光子荧光成像研究.

关键词 溶酶体; 亚硝酰氢; 双光子; 细胞成像
\end{abstract}

\section{Lysosome-Targeted Dual-Photon Nitroxyl Fluorescent Probe: Synthesis and Application in Living Cell Imaging}

\author{
Wang, Xiaofen Wei, Chao* Li, Xueyan Zheng, Xueyang \\ Geng, Xiaowei Zhang, Pingzhu Li, Xiaoliu*
}

(Key Laboratory of Medicinal Chemistry and Molecular Diagnosis of Ministry of Education, Key Laboratory of Chemical Biology of Hebei Province, College of Chemistry and Environmental Science, Hebei University, Baoding 071002)

\begin{abstract}
Nitroxyl (HNO), which is the one-electron reduced and further protonated form of nitric oxide, plays important biological functions. A lysosome-targeted dual-photon HNO fluorescent probe (Lyso-HNO), which contains 4-(2-aminoethyl)morpholine as lysosomal-targetable groups, 1,8-naphthalimide as two-photon fluorophore and triphenylphosphine as HNO reaction site, was synthesized and characterized. The recognition behaviors of Lyso-HNO to HNO were investigated. The results showed that Lyso-HNO exhibited good selectivity and sensitivity to HNO with fast response,. and the detection limit of Lyso-HNO to HNO was estimated to be $202 \mathrm{nmol} \cdot \mathrm{L}^{-1}$. The probe can be applied to bioimaging exogenous lysosomal HNO by two-photon fluorescence confocal microscopy.

Keywords lysosome; dual-photon; nitroxyl; bioimaging
\end{abstract}

亚硝酰氢( $\mathrm{HNO})$ 是一氧化氮(NO)单电子还原并质 子化的产物, 生命体内可以由一氧化氮合成酶(NOS)氧 化降解 $L$-精氨酸而成. HNO 具有独特的化学性质, 可以 与生命体内的颈基化合物、金属离子以及金属蛋白发生 相互作用, 在多种生理和病理过程中发挥着重要的作 用 ${ }^{[1 \sim 3]}$. 自从被发现以来, HNO 在调节心血管系统、免疫 系统和神经系统等方面引起研究者的广泛关注 ${ }^{[4 \sim 6]}$. 此 外, HNO 还可以刺激心血管舒张和抑制乙醛脱氢酶活
性，已经作为治疗心脏衰竭和酗酒的潜在药物 ${ }^{[7,8]}$.

溶酶体被称为细胞的 “清道夫” , 是细胞正常生长 所需的重要细胞器, 可以通过自噬过程调节细胞的新陈 代谢 ${ }^{[9]}$. 研究表明, NO 可以与溶酶体外部相关蛋白发生 相互作用，进而引发溶酶体的自噬过程 ${ }^{[10]}$. 在超氧化物 歧化酶催化下, HNO 可以与 NO 相互转化 ${ }^{[11]}$. 因此, 开 发可视化溶酶体内 HNO 的荧光方法对进一步研究 HNO 在溶酶体自噬过程中的作用具有重要意义.

\footnotetext{
* Corresponding authors. E-mail: weichao@hbu.edu.cn; lixl@hbu.edu.cn.

Received July 20, 2018; revised August 27, 2018; published online September 17, 2018.

Project supported by the National Natural Science Foundation of China (Nos. 21572044, 21778013), the Natural Science Foundation of Hebei Provience (Nos. B2016201031, 2018201234), the Colleges and Universities Science Technology Research Project of Hebei Province (No. QN2017015) and the Science Technology Research and Development Guidance Program Project of Baoding City (No. 16zg031).

国家自然科学基金(Nos. 21572044, 21778013)、河北省自然科学基金(Nos. B2016201031, 2018201234)、河北省高等学校科学技术研究(No. QN2017015) 和保定市科学研究与发展计划(No. 16zg031)资助项目.
} 
目前, 多种检测 HNO 的荧光探针被报道, 这些探 针主要基于三种识别原理 ${ }^{[12,13]}$ : $\mathrm{HNO}$ 将 $\mathrm{Cu}(\mathrm{II})$ 还原成 $\mathrm{Cu}(\mathrm{I})^{[14,15]}, \mathrm{HNO}$ 将氮氧化合物还原成羟胺 ${ }^{[16]}$; HNO 与三 苯基膦发生施陶丁格反应(Staudinger) ${ }^{[17 \sim 26,28 ~ 30]}$. 虽然 这些荧光探针能够选择性检测 HNO, 但是大部分探针 属于单光子激发的荧光探针 ${ }^{[17 ~ 26]}$. 相比于单光子激发, 双光子激发的苂光探针使用近红外光作为激发光源, 具 有长波长激发、较低的自发荧光干扰、较小的光损伤、 较少的光漂白和较深的组织穿透深度等优点 ${ }^{[28-30]}$. 1,8荎酰亚胺是一种经典的苂光染料 ${ }^{[31 \sim 34]}$, 它具有典型的 电子供体- $\pi$-电子受体 $(D-\pi-A)$ 双光子荧光染料结构, 具 有易于合成、光稳定性好、斯托克斯位移大等特点, 已 经被广泛用于组织深层次双光子成像 ${ }^{[35 \sim 37]}$.

本文以 4-(2-氨基乙基)-吗啉为溶酶体靶向基团, 1,8-萗二甲酰亚胺为双光子荧光团, 三苯基膦作为 HNO 识别基团, 经过 4 步简单有机合成, 得到一个能够特异 性定位于溶酶体的打开型双光子 HNO 苂光探针(Lyso$\mathrm{HNO}$ ), 测试了探针对 HNO 的选择性和灵敏性, 研究了 探针对细胞溶酶体内 $\mathrm{HNO}$ 的共聚焦成像.<smiles>O=C(O)c1ccc(-c2ccccc2)cc1C(=O)OCCN1C(=O)c2cccc3c(O)ccc(c23)C1=O</smiles><smiles>O=C(Oc1ccc2c3c(cccc13)C(=O)N(CCN1CCOCC1)C2=O)c1ccccc1-c1ccccc1</smiles>

\section{1 结果与讨论}

\section{1 荧光探针 Lyso-HNO 的设计}

$\mathrm{HNO}$ 是生命体内重要的活性物种, 参与多种生命 调节过程. 当前报道的 HNO 苂光探针可以用于检测细 胞内 HNO, 但是它们多为单光子激发的苂光探针. 探针 Lyso-HNO 以萗酰亚胺作为双光子激发荧光染料, 这种 近红外光作为激发光源在降低光损伤和光漂白方面更 具有优势, 更利于活细胞荧光成像. 同时, 探针 Lyso-HNO 具有溶酶体定位功能, 能够用于溶酶体内 HNO 的共聚焦成像研究.

\section{2 荧光探针 Lyso-HNO 的光谱性质研究}

\subsection{1 荧光探针 Lyso-HNO 的荧光反应动力学研究}

由于 HNO 不稳定, 易脱水发生不可逆的二聚化反 应, 因此, 快速响应型探针更能准确检测 HNO 的实际 浓度. 溶酶体内 $\mathrm{pH}$ 在 $4.0 \sim 6.0$ 范围内 ${ }^{[38]}$, 选取磷酸缓冲
盐溶液(PBS) $\left(10 \mathrm{mmol} \cdot \mathrm{L}^{-1}, \mathrm{pH} 5.0,10 \% \mathrm{~N}, \mathrm{~N}\right.$-二甲基甲 酰胺), 测试了室温下探针对 HNO 的响应速度. 如图 1 所示, 向探针溶液 $\left(5 \mu \mathrm{mol} \cdot \mathrm{L}^{-1}\right)$ 中加入 $\mathrm{HNO}\left(50 \mu \mathrm{mol} \cdot \mathrm{L}^{-}\right.$ $\left.{ }^{1}\right)$, 溶液出现明显的黄绿色苂光, $2 \mathrm{~min}$ 后反应体系的苂 光强度基本饱和. 利用 $570 \mathrm{~nm}$ 处荧光强度 $\left(I_{570} \mathrm{~nm}\right)$ 对反 应时间 $(t)$ 作图, 根据公式计算 ${ }^{[24]}$, 探针的准一级反应动 力学常数 $K_{\mathrm{obs}}$ 、二级反应动力学常数 $K_{2}$ 、半衰期 $t_{1 / 2}$ 分 别为 $2.59 \times 10^{-3} \mathrm{~s}^{-1} 、 517.4 \mathrm{~mol} \cdot \mathrm{L}^{-1} \cdot \mathrm{s}^{-1}$ 和 $27 \mathrm{~s}$, 说明探 针 Lyso-HNO 响应快速, 具有实时检测 HNO 的能力.
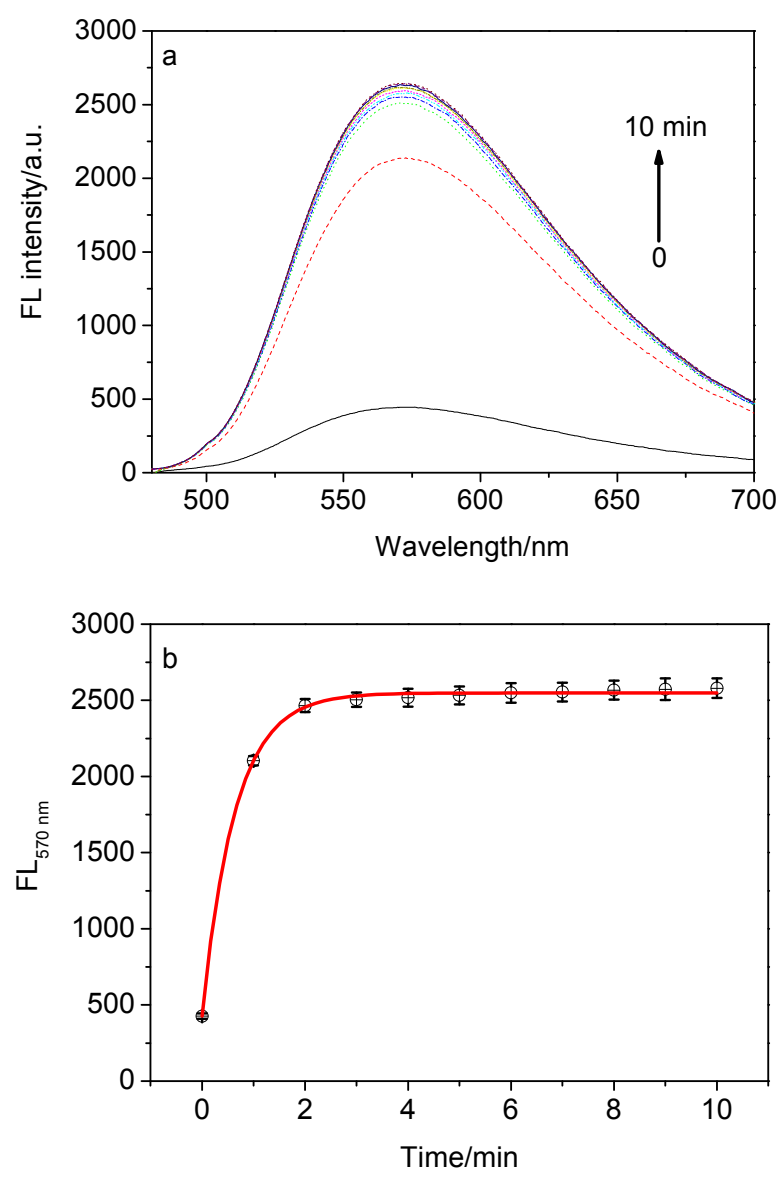

图 1 (a)探针 Lyso-HNO 加入 HNO 后随时间变化的苂光发射 光谱和(b)探针 Lyso-HNO 与 HNO 反应后 $570 \mathrm{~nm}$ 处荧光随时 间的变化曲线(Lyso-HNO $5 \mu \mathrm{mol} \cdot \mathrm{L}^{-1}, \mathrm{HNO} 50 \mu \mathrm{mol} \cdot \mathrm{L}^{-1}, E_{\mathrm{x}}$ $450 \mathrm{~nm}$ )

Figure 1 (a) Time dependence of fluorescence spectra of Lyso-HNO with HNO, and (b) time dependence of fluorescence intensity of Lyso-HNO at $570 \mathrm{~nm}$ with HNO (Lyso-HNO 5 $\mu \mathrm{mol} \cdot \mathrm{L}^{-1}$, HNO $\left.50 \mu \mathrm{mol} \cdot \mathrm{L}^{-1}, E_{\mathrm{x}} 450 \mathrm{~nm}\right)$

\subsection{2 荧光探针 Lyso-HNO 的选择性研究}

高度专一的选择性是性能优良荧光探针的必备条 件, 生物体系中存在多种类型的活性物种. 为了考察探 针的选择性，测试了探针对各种活性氧、活性氮、颈基 化合物以及其它相关分析物的苂光光谱响应，这些分析 物包括，还原型分析物 $\left(\mathrm{Na}_{2} \mathrm{~S} 、 \mathrm{NaNO}_{2} 、 \mathrm{NO}\right)$ 、氧化型分 
析物 $\left(\mathrm{H}_{2} \mathrm{O}_{2} 、 \mathrm{NaClO} 、 \mathrm{KO}_{2} 、 \mathrm{TBHP}\right)$ 、颈基化合物( $\mathrm{GSH}$ 、 Cys、Hcy) 和金属离子 $\left(\mathrm{Na}^{+} 、 \mathrm{Ca}^{2+} 、 \mathrm{Cu}^{2+} 、 \mathrm{Fe}^{3+} 、 \mathrm{Mg}^{2+} 、\right.$ $\mathrm{Zn}^{2+}$ ). 如图 2 所示, 向探针溶液中加入其它分析物并没 有使探针溶液的荧光强度发生明显变化, 而加入 $\mathrm{HNO}$ 使探针溶液在 $570 \mathrm{~nm}$ 处的荧光强度显著增强. 说明探 针 Lyso-HNO 对 HNO 的识别具有较强的专一性, 也表 明该探针在生物系统中具有潜在的应用价值.

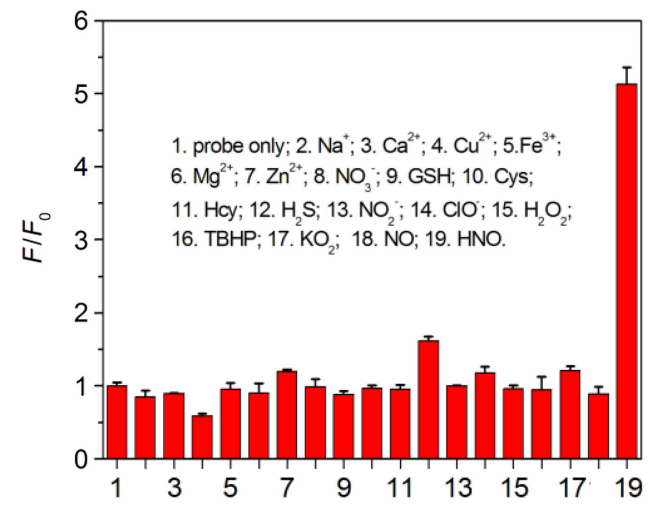

图 2 探针 Lyso-HNO $\left(5 \mu \mathrm{mol} \cdot \mathrm{L}^{-1}\right)$ 加入各种分析物后在 570 $\mathrm{nm}$ 处的苂光响应图

Figure 2 Fluorescence enhancement at $570 \mathrm{~nm}$ of Lyso-HNO $\left(5 \mu \mathrm{mol} \cdot \mathrm{L}^{-1}\right)$ upon individual addition of different species

\subsection{3 荧光探针 Lyso-HNO 的灵敏性研究}

为了考察探针的灵敏性, 测试了探针与不同浓度 $\mathrm{HNO}$ 反应后的苂光光谱图. 如图 3 所示, 随着 HNO 浓 度逐渐增大, 探针溶液在 $570 \mathrm{~nm}$ 处的苂光强度逐渐增 强, 当加入 $\mathrm{HNO}$ 浓度高于 $50 \mu \mathrm{mol} \cdot \mathrm{L}^{-1}$ 时, 探针苂光强 度基本保持不变. 根据滴定实验发现, 探针溶液的苂光 强度与 HNO 浓度 $\left(0 \sim 25 \mu \mathrm{mol} \cdot \mathrm{L}^{-1}\right)$ 具有较好的线性关系 (线性相关系数: $R^{2}=0.99838$ ), 表明探针可以用来定量 检测 HNO. 根据 $3 \sigma / \mathrm{k}$ 的计算方法 $(\sigma$ 是空白溶液的标准 偏差, $\mathrm{k}$ 是荧光强度与 $\mathrm{HNO}$ 浓度线性关系的斜率 $)^{[39]}$, 探 针的检测极限为 $202 \mathrm{nmol} \cdot \mathrm{L}^{-1}$. 以上结果表明, 探针 Lyso-HNO 可以用于低浓度 HNO 检测.

\subsection{4 荧光探针 Lyso-HNO 的识别机理研究}

为了验证探针的识别机理, 测试了探针与 HNO 反 应后的紫外吸收光谱和高分辨质谱. 如图 4 所示, 探针 在 $350 \mathrm{~nm}$ 左右处有一个强吸收带. 随着反应的进行, $350 \mathrm{~nm}$ 处的吸收峰强度逐渐减弱, $450 \mathrm{~nm}$ 处的吸收峰逐 渐增强, 这是由于探针结构中萗酰亚胺 4 位从酯基变为 羟基, 给电子能力增强, 紫外吸收峰红移. 通过高分辨 质谱发现, 探针与 HNO 反应后, 出现 327.1342 分子离 子峰, 与化合物 3 的理论分子量相符 $\left([\mathrm{M}+\mathrm{H}]^{+}=\right.$ 327.1345). 以上结果表明, 探针结构中的三苯基膦基团 与 $\mathrm{HNO}$ 发生 Staudinger 反应生成氮叶立德中间体, 接着 氮叶立德中间体与分子内酯基发生亲核反应, 形成二苯
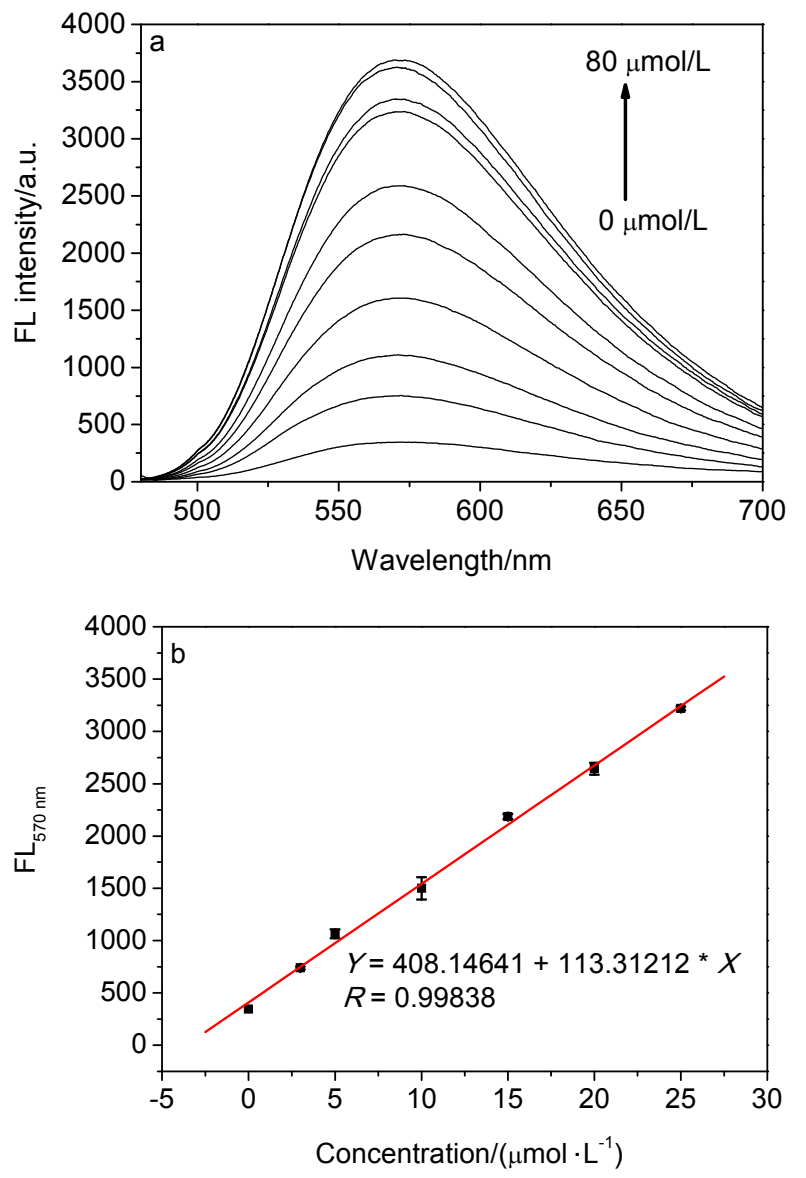

图 3 (a)探针 Lyso-HNO $\left(5 \mu \mathrm{mol} \cdot \mathrm{L}^{-1}\right)$ 在不同浓度 HNO $(0 \sim 80$ $\mu \mathrm{mol} \cdot \mathrm{L}^{-1}$ ) 中的苂光光谱图和(b)探针在 $570 \mathrm{~nm}$ 处的荧光强度 与 $\mathrm{HNO}$ 浓度 $\left(0 \sim 25 \mu \mathrm{mol} \cdot \mathrm{L}^{-1}\right)$ 的线性关系

Figure 3 (a) Fluorescence spectra of Lyso-HNO $\left(5 \mu \mathrm{mol} \cdot \mathrm{L}^{-1}\right)$ in the presence of HNO $\left(0 \sim 80 \mu \mathrm{mol} \cdot \mathrm{L}^{-1}\right)$, and (b) the linear relationship between the fluorescence intensity $\left(I_{570 \mathrm{~nm}}\right)$ and the concentration of $\mathrm{HNO}\left(0 \sim 25 \mu \mathrm{mol} \cdot \mathrm{L}^{-1}\right)$

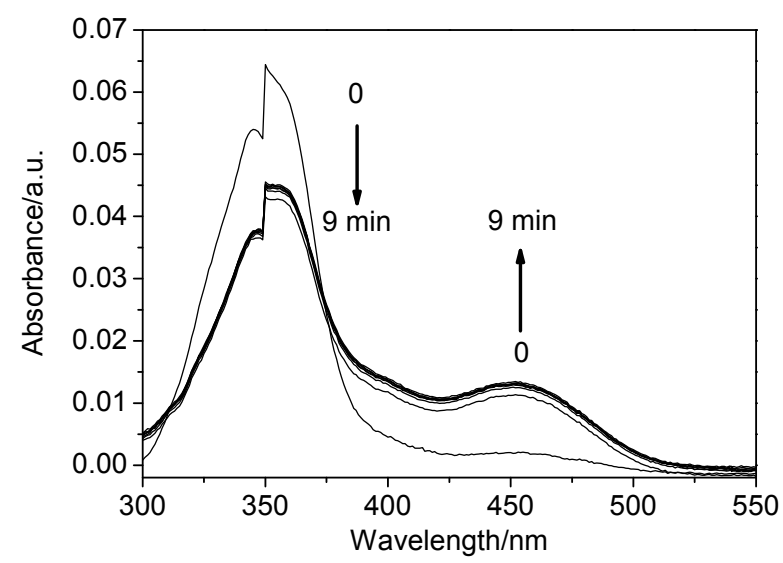

图 4 探针 $\mathrm{Lyso}-\mathrm{HNO}\left(5 \mu \mathrm{mol} \cdot \mathrm{L}^{-1}\right)$ 加入 $\mathrm{HNO}\left(50 \mu \mathrm{mol} \cdot \mathrm{L}^{-1}\right)$ 后 随时间变化的紫外吸收光谱图

Figure $4 \mathrm{UV}$-Vis spectra of Lyso-HNO $\left(5 \mu \mathrm{mol} \cdot \mathrm{L}^{-1}\right)$ upon addition of $\mathrm{HNO}\left(50 \mu \mathrm{mol} \bullet \mathrm{L}^{-1}\right)$ at different incubation time

基膦酰基苯甲酰胺，释放出化合物 $\mathbf{3}$, 最终使苂光团恢 
复发射荧光. 其机理如 Scheme 1 所示.

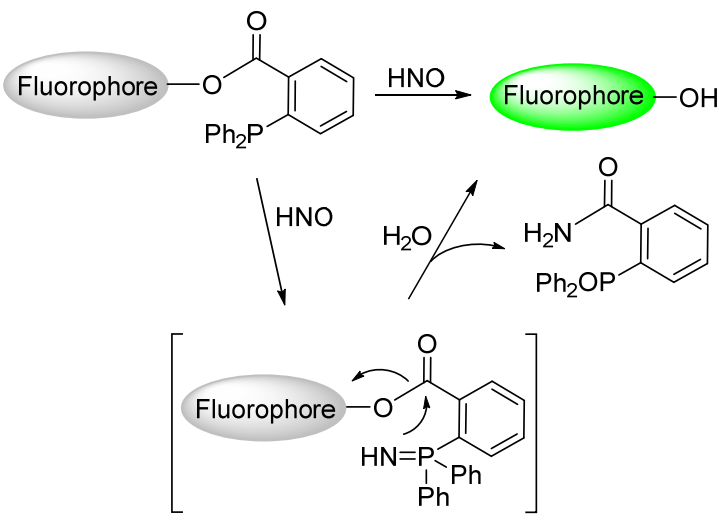

图式 1 探针 Lyso-HNO 对 HNO 的识别机理

Scheme 1 Sensing mechanism of Lyso-HNO to HNO

\subsubsection{HeLa 细胞成像研究}

为了进一步研究探针在活细胞中对 HNO 响应的能 力, 首先利用噻唑蓝(MTT)法对探针进行了细胞毒性测 试, 选取宫颈癌 HeLa 细胞和人正常肾 HEK293 细胞两 种细胞株. 实验结果表明, 探针在 $0 \sim 20 \mu \mathrm{mol} \cdot \mathrm{L}^{-1}$ 浓度 范围内对测试细胞株均未表现出明显的抑制作用, 说明 探针适用于在活细胞中对 HNO 进行检测. 据文献报道, 吗啉结构可以定位于细胞中的溶酶体细胞器, 测试了探

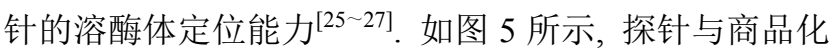
溶酶体定位探针 Lyso-Tracker Red 共孵育后, 共定位能 力良好, 皮尔森相关系数 (Pearson's correlation coefficient)为 0.93 , 表明探针可以定位于溶酶体细胞器. 菜酰 亚胺荧光团是一种双光子吸收荧光团, 探针被应用到 HeLa 细胞的双光子荧光成像实验. 如图 6 所示, 探针单 独孵育的 $\mathrm{HeLa}$ 细胞基本没有绿色荧光(图 6A); 当加 入 $\mathrm{HNO}$ 继续孵育后, 绿色荧光明显增强(图 6B). 这 些结果表明探针 Lyso-HNO 具有良好的细胞渗透性, 可以用来检测活细胞溶酶体内的 HNO.

\section{2 结论}

设计合成了一种溶酶体靶向荧光增强型双光子 HNO 苂光探针 Lyso-HNO, 通过 HNO 引发的 Staudinger 反应, 释放前体化合物 3. 探针对 HNO 表现出良好的选 择性和灵敏性, 并且响应迅速, 检测极限为 $202 \mathrm{nmol}$ $\mathrm{L}^{-1}$. 此外, 探针具有双光子吸收功能和溶酶体定位能 力, 可用于活细胞溶酶体中 $\mathrm{HNO}$ 的检测, 具有潜在的 应用价值.

\section{3 实验部分}

\section{1 仪器与试剂}

Brucker 核磁共振仪 $(400 \mathrm{MHz}$ 和 $600 \mathrm{MHz})$; Varian
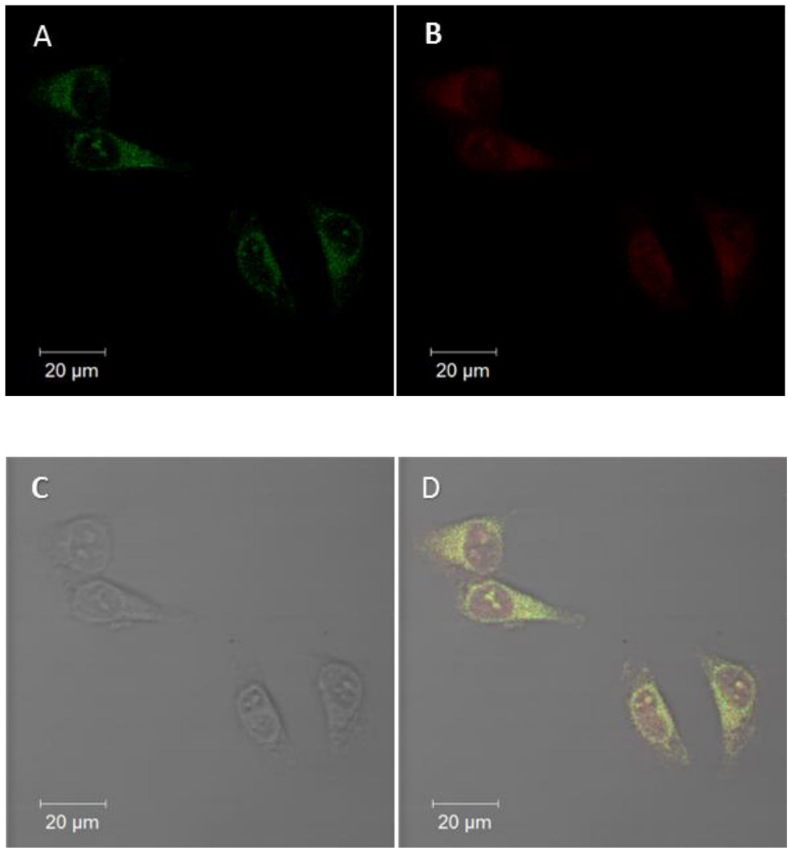

图 5 探针 Lyso-HNO 溶酶体苂光共定位成像 $\mathrm{A}$ 为探针

Figure 5 Colocalization imaging of lysosomes in HeLa cells using Lyso-HNO

(A): Fluorescence image of Lyso-HNO; (B): Fluorescence image of Lyso-Tracker Red; 3: bright-field image; 4: Merged images of A, B and C; Scale bars $=20 \mu \mathrm{m}$ )

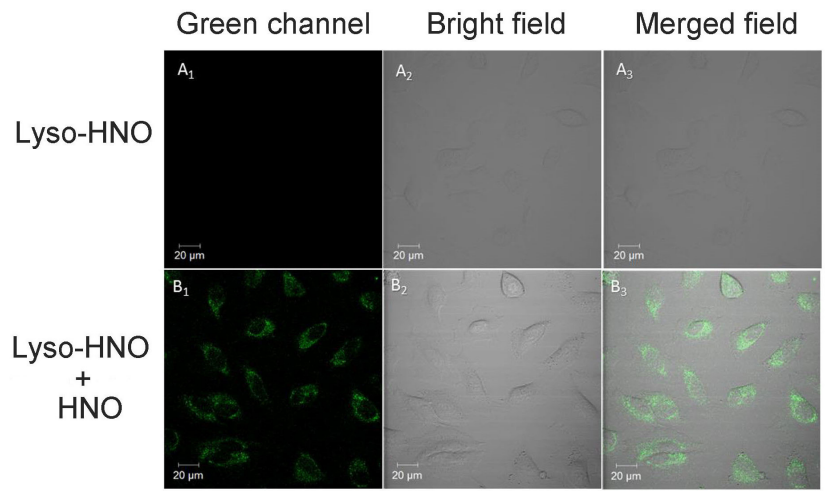

图 6 探针 Lyso-HNO 培养的 HeLa 细胞双光子苂光共聚焦成 像研究

Figure 6 Two-photon confocal fluorescence imaging of exogenous $\mathrm{HNO}$ in HeLa cells using Lyso-HNO

(A) Incubation probe $\left(5 \mu \mathrm{mol} \cdot \mathrm{L}^{-1}\right)$ only; (B) incubation probe $(5 \mu \mathrm{mol} \cdot$ $\left.\mathrm{L}^{-1}\right)$ firstly, then $\mathrm{HNO}\left(50 \mu \mathrm{mol} \cdot \mathrm{L}^{-1}\right)$. 1: fluorescence images, 2: brightfield images, 3: Merged images of 1 and 2; Scale bars $=20 \mu \mathrm{m}$

7.0 T FTICR-MS 质谱仪(美国 Agilent 公司); HitachiF7000 型苂光光谱仪(日本 Hitachi 公司); UV-3600 型分光 光度计(美国 Agilent 公司).

柱层析用硅胶(100 200 目)及 GF254 薄层层析硅 胶为青岛海洋化工厂产品; 所用试剂和溶剂均为国产或 进口, 分析纯或者化学纯试剂, 无水试剂均按常规方法 处理. 

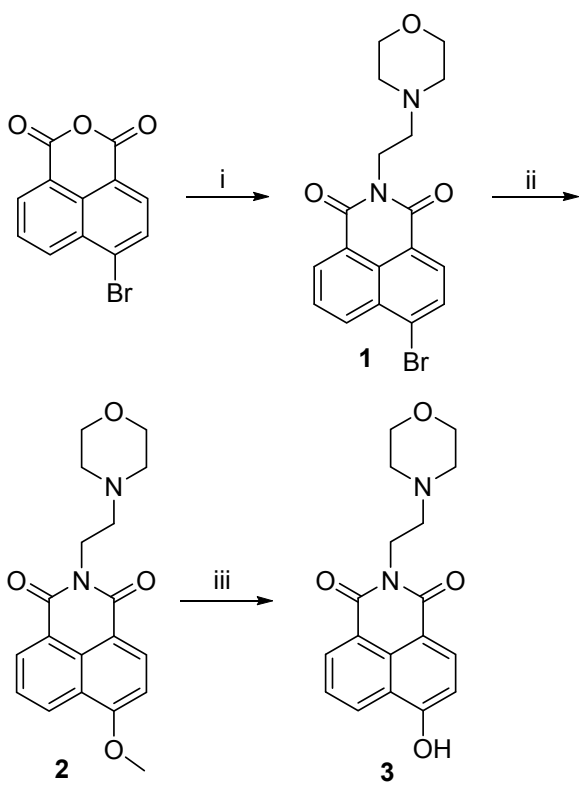

Reagents and conditions: (i) $\mathrm{N}$-(2-aminoethyl)morpholine, $\mathrm{EtOH}$, reflux, $5 \mathrm{~h}$; (ii) $\mathrm{CH}_{3} \mathrm{OH}, \mathrm{K}_{2} \mathrm{CO}_{3}$, reflux, $24 \mathrm{~h}$; (iii) $\mathrm{HI}$, reflux, $5 \mathrm{~h}$.

图式 2 探针 Lyso-HNO 的合成

Scheme 2 Synthesis of probe Lyso-HNO

\section{2 实验方法}

\section{2 .1 化合物 $1 、 2$ 和 3 的合成}

向 $100 \mathrm{~mL}$ 圆底烧瓶中加入 $5.0 \mathrm{~g}(18.0 \mathrm{mmol}) 4$-澳1,8-菜二酸酐, $5.0 \mathrm{~g}(36.0 \mathrm{mmol})$ 胺乙基吗啉和 $50 \mathrm{~mL}$ 无 水乙醇, 回流反应 $8 \mathrm{~h}$. 将反应液冷却至室温, 析出沉 淀, 抽滤, 少量无水乙醇洗涤沉淀, 干燥, 得到 $6.2 \mathrm{~g}$ 淡 黄色固体 1, 产率 $90 \%$. m.p. $165.2 \sim 166.8{ }^{\circ} \mathrm{C} ;{ }^{1} \mathrm{H}$ NMR $\left(400 \mathrm{MHz}, \mathrm{CDCl}_{3}\right) \delta: 8.65(\mathrm{~d}, J=7.2 \mathrm{~Hz}, 1 \mathrm{H}), 8.57(\mathrm{~d}, J=$ $8.4 \mathrm{~Hz}, 1 \mathrm{H}), 8.40(\mathrm{~d}, J=7.6 \mathrm{~Hz}, 1 \mathrm{H}), 8.04(\mathrm{~d}, J=8.0 \mathrm{~Hz}$, $1 \mathrm{H}), 7.85(\mathrm{t}, J=8.0 \mathrm{~Hz}, 1 \mathrm{H}), 4.34(\mathrm{t}, J=6.4 \mathrm{~Hz}, 2 \mathrm{H}), 3.68$ (s, 4H), 2.71 (t, $J=6.4 \mathrm{~Hz}, 2 \mathrm{H}), 2.61$ (s, 4H). HRMS calcd for $\mathrm{C}_{18} \mathrm{H}_{18} \mathrm{BrN}_{2} \mathrm{O}_{3} 389.0501$, found 389.0492 .

向 $50 \mathrm{~mL}$ 圆底烧瓶中加入 $2.5 \mathrm{~g}(6.40 \mathrm{mmol})$ 化合物 $1,3.50 \mathrm{~g}(25.3 \mathrm{mmol})$ 碳酸钾和 $30 \mathrm{~mL}$ 无水甲醇, 回流反 应 $24 \mathrm{~h}$. 将反应液冷却至室温, 析出沉淀, 抽滤, 少量 水洗涤沉淀, 干燥, 得到 $1.6 \mathrm{~g}$ 淡黄色针状固体 2 , 产率 76 \%. m.p. $125.5 \sim 127.0{ }^{\circ} \mathrm{C} ;{ }^{1} \mathrm{H}$ NMR $\left(400 \mathrm{MHz}, \mathrm{CDCl}_{3}\right)$ $\delta: 8.59 \sim 8.53(\mathrm{~m}, 3 \mathrm{H}), 7.70(\mathrm{t}, J=7.6 \mathrm{~Hz}, 1 \mathrm{H}), 7.04(\mathrm{~d}$, $J=8.0 \mathrm{~Hz}, 1 \mathrm{H}), 4.34$ (t, $J=7.2 \mathrm{~Hz}, 2 \mathrm{H}), 4.13(\mathrm{~s}, 3 \mathrm{H}), 3.70$ (t, $J=4.8 \mathrm{~Hz}, 4 \mathrm{H}), 2.71(\mathrm{t}, J=7.2 \mathrm{~Hz}, 2 \mathrm{H}), 2.62(\mathrm{~s}, 4 \mathrm{H})$. HRMS calcd for $\mathrm{C}_{19} \mathrm{H}_{21} \mathrm{~N}_{2} \mathrm{O}_{4} 341.1501$, found 341.1497.

向 $50 \mathrm{~mL}$ 圆底烧瓶中加入 $1.0 \mathrm{~g}(3.00 \mathrm{mmol})$ 化合物 2 和 $50 \mathrm{~mL} 57 \% \mathrm{HI}$, 回流反应 $6 \mathrm{~h}$. 将反应液冷却至室 温, 用 $2 \mathrm{~mol} \cdot \mathrm{L}^{-1} \mathrm{NaOH}$ 水溶液调节 $\mathrm{pH}$ 至中性, 有淡黄 色针状沉淀生成. 抽滤, 少量无水乙醇洗涤沉淀, 干燥, 得到 $0.82 \mathrm{~g}$ 淡黄色针状固体 3, 产率 $86 \%$. m.p. 116.3
$117.8{ }^{\circ} \mathrm{C} ;{ }^{1} \mathrm{H}$ NMR (600 MHz, DMSO- $\left.d_{6}\right) \delta: 8.55$ (d, $J=$ $6.4 \mathrm{~Hz}, 1 \mathrm{H}), 8.48(\mathrm{~d}, J=7.2 \mathrm{~Hz}, 1 \mathrm{H}), 8.35(\mathrm{~d}, J=8.4 \mathrm{~Hz}$, $1 \mathrm{H}), 7.76(\mathrm{t}, J=8.4 \mathrm{~Hz}, 1 \mathrm{H}), 7.14(\mathrm{~d}, J=7.8 \mathrm{~Hz}, 1 \mathrm{H}), 4.17$ (t, $J=7.2 \mathrm{~Hz}, 2 \mathrm{H}), 3.54$ (t, $J=4.2 \mathrm{~Hz}, 4 \mathrm{H}), 2.57$ (t, $J=7.2$ $\mathrm{Hz}, 2 \mathrm{H}), 2.49$ (s, 4H). HRMS calcd for $\mathrm{C}_{18} \mathrm{H}_{19} \mathrm{~N}_{2} \mathrm{O}_{4}$ 327.1345 , found 327.1335 .

\subsection{2 探针 Lyso-HNO 的合成}

将 $200 \mathrm{mg}(0.61 \mathrm{mmol})$ 化合物 3 溶解于 $15 \mathrm{~mL}$ 无水 二氯甲烷, 依次加入 $151 \mathrm{mg}(0.73 \mathrm{mmol})$ 二环己基碳二 亚胺、 $224 \mathrm{mg}(0.73 \mathrm{mmol}) 2$-二苯基膦苯甲酸和 $89 \mathrm{mg}$ (0.73 mmol) 4-二甲氨基吡啶, 室温摚拌过夜. 将反应液 浓缩, 柱层析分离 $[V$ (二氯甲烷) $: V$ (乙酸乙酯 $)=4: 1]$, 得到 $354 \mathrm{mg}$ 白色固体 Lyso-HNO, 产率 79\%. m.p. $220.5 \sim 221.9{ }^{\circ} \mathrm{C} ;{ }^{1} \mathrm{H}$ NMR $\left(400 \mathrm{MHz}, \mathrm{CDCl}_{3}\right) \delta: 8.59 \sim$ $8.53(\mathrm{~m}, 2 \mathrm{H}), 8.45 \sim 8.42(\mathrm{~m}, 1 \mathrm{H}), 8.05(\mathrm{~d}, J=8.0 \mathrm{~Hz}$, $1 \mathrm{H}), 7.65(\mathrm{t}, J=8.0 \mathrm{~Hz}, 1 \mathrm{H}), 7.58 \sim 7.53(\mathrm{~m}, 2 \mathrm{H}), 7.38 \sim$ $7.27(\mathrm{~m}, 11 \mathrm{H}), 7.10 \sim 7.07(\mathrm{~m}, 1 \mathrm{H}), 4.34(\mathrm{t}, J=6.8 \mathrm{~Hz}$, $2 \mathrm{H}), 3.68(\mathrm{t}, J=4.4 \mathrm{~Hz}, 2 \mathrm{H}), 2.71(\mathrm{t}, J=6.8 \mathrm{~Hz}, 2 \mathrm{H}), 2.68$ $(\mathrm{s}, 4 \mathrm{H}) ;{ }^{13} \mathrm{C}$ NMR $\left(125 \mathrm{MHz}, \mathrm{CDCl}_{3}\right) \delta: 164.38,164.01$, $163.51,151.54,142.57,137.20,137.13,134.65,134.11$, $133.97,133.20,131.68,131.51,129.31,128.95,128.67$, $128.62,128.48,128.00,127.09,125.33,122.72,120.32$, 119.50, 67.03, 56.12, 53.80, 37.20. HRMS $\left(\mathrm{ESI}^{+}\right)$calcd for $\mathrm{C}_{37} \mathrm{H}_{32} \mathrm{~N}_{2} \mathrm{O}_{5} 615.2043$, found 615.2045.

\subsection{3 光谱测试}

探针 Lyso-HNO 用二甲基亚砜(DMSO)配制成 5 $\mathrm{mmol} \cdot \mathrm{L}^{-1}$ 的母液, 测试浓度为 $5 \mu \mathrm{mol} \cdot \mathrm{L}^{-1}$. 选择性测试 所用各种干扰离子均为钠盐或钾盐, 除谷胱甘肽(GSH) 为 $5 \mu \mathrm{mmol} \cdot \mathrm{L}^{-1}$ 外, 其它干扰离子测试浓度为 $500 \mu \mathrm{mol} \cdot$ $\mathrm{L}^{-1}$, 测试溶液为 $\mathrm{PBS}$ 缓冲溶液 $\left(10 \mathrm{mmol} \cdot \mathrm{L}^{-1}, \mathrm{pH} 5.0\right.$, $10 \%$ DMF). Angeli's salt (HNO 供体)和硝普化钠(NO 供 体) 溶解在 $0.01 \mathrm{~mol} \cdot \mathrm{L}^{-1} \mathrm{NaOH}$ 中, $\mathrm{KO}_{2}$ 溶解在 DMSO 中 制备所需浓度的母液. 苂光光谱均在室温条件下测试, 样品池为 $1 \mathrm{~cm} \times 1 \mathrm{~cm} \times 4 \mathrm{~cm}$ 石英比色血, 激发波长为 $450 \mathrm{~nm}$, 激发和发射狭缝宽度分别为 5 和 $10 \mathrm{~nm}$.

\subsection{4 细胞实验}

$\mathrm{HeLa}$ 细胞培养条件：细胞培养基为高糖培养基, 其中含有体积比为 $10 \%$ 胎牛血清、 $100 \mathrm{U} / \mathrm{mL}$ 盘尼西林、 $100 \mathrm{mg} / \mathrm{mL}$ 链霉素和 $4 \mathrm{mM} \mathrm{L}$-谷氨酰胺. $\mathrm{HeLa}$ 细胞在体 积分数为 $5 \% \mathrm{CO}_{2}$ 的环境下培养, 待生长至融合度为 $70 \% \sim 80 \%$ 时，加入质量分数为 $0.25 \%$ 的胰酶蛋白消化 细胞进行实验操作.

细胞毒性实验: 将 HeLa 细胞接种于 96 孔板, 每孔 细胞浓度为 $10^{4}$, 过夜孵育后, 加入不同浓度的 Lyso$\mathrm{HNO}\left(0,1,2,5,10,15,20 \mu \mathrm{mol} \cdot \mathrm{L}^{-1}\right)$, 继续粰育 $3 \mathrm{~h}$. 向 
每孔加入四甲基偶氮唑盐(MTT), 终浓度为 $0.5 \mathrm{mg} / \mathrm{mL}$, 继续孵育 $4 \mathrm{~h}$. 用 $150 \mu \mathrm{L} \mathrm{DMSO}$ 溶解生成的甲珗沉淀, 通过酶标仪在 $490 \mathrm{~nm}$ 处检测其光密度(OD)值.

细胞共定位成像实验: HeLa 细胞接种于 $35 \mathrm{~mm}$ 细 胞成像专用玻底培养血, 成像细胞密度为 $2 \times 10^{4}$. 首先 加入 $\mathrm{HNO}\left(50 \mu \mathrm{mol} \cdot \mathrm{L}^{-1}\right)$ 孵育 $30 \mathrm{~min}$, 再加入 Lyso-HNO $\left(5 \mu \mathrm{mol} \cdot \mathrm{L}^{-1}\right)$ 和商品化溶酶体定位探针 Lyso-Tracker Red $\left(20 \mathrm{nmol} \cdot \mathrm{L}^{-1}\right)$ 共孵育 $30 \mathrm{~min}$, 用 $\mathrm{PBS}$ 洗涤三次后, 进行 共定位成像研究. 探针 Lyso-HNO 苂光通道激发波长为 $780 \mathrm{~nm}$, 收集 500 550 nm 范围; Lyso-Tracker Red 荧光 通道激发波长为 $546 \mathrm{~nm}$, 收集 590 640 nm 范围.

细胞外源 HNO 成像实验: 单独加入 Lyso-HNO (5 $\mu \mathrm{mol} \cdot \mathrm{L}^{-1}$ )孵育 $30 \mathrm{~min}$, 用 $\mathrm{PBS}$ 洗涤三次后, 采集明场和 荧光场图像, 进行细胞成像研究; 首先加入 Lyso-HNO $\left(5 \mu \mathrm{mol} \cdot \mathrm{L}^{-1}\right)$ 孵育 $30 \mathrm{~min}$, 用 $\mathrm{PBS}$ 洗涤三次后, 加入 HNO $\left(50 \mu \mathrm{mol} \cdot \mathrm{L}^{-1}\right)$, 继续孵育 $45 \mathrm{~min}$, 最后采集明场和 荧光场图像, 进行细胞成像研究. 探针 Lyso-HNO 苂光 通道激发波长为 $780 \mathrm{~nm}$, 收集 500 $550 \mathrm{~nm}$ 范围.

辅助材料(Supporting Information) 所有化合物的 ${ }^{1} \mathrm{H}$ $\mathrm{NMR},{ }^{13} \mathrm{C}$ NMR 以及 HRMS 谱图, 探针的毒性, 探针与 $\mathrm{HNO}$ 反应后的高分辨质谱. 这些材料可以免费从本刊 网站(http://sioc-journal.cn/)上下载.

\section{References}

[1] Paolocci, N.; Jackson, M. I.; Lopez, B. E.; Miranda, K.; Tocchetti, C. G.; Wink, D. A.; Hobbs, A. J.; Fukuto, J. M. Pharmacol. Therapeut. 2007, 113, 442 .

[2] Irvine, J. C.; Ritchie, R. H.; Favaloro, J. L.; Andrews, K. L.; Widdop, R. E.; Kemp-Harper, B. K. Trends Pharmacol. Sci. 2008, 29, 601 .

[3] Flores-Santana, W.; Salmon, D. J.; Donzelli, S.; Switzer, C. H.; Basudhar, D.; Ridnour, L.; Cheng, R.; Glynn, S. A.; Paolocci, N.; Fukuto, J. M.; Miranda, K. M.; Wink, D. A. Antioxid. Redox Sign. 2011, 14, 1659.

[4] Paolocci, N.; F. Saavedra, W.; Miranda, K.; Martignani, C.; Isoda, T.; M. Hare, J.; G. Espey, M.; M. Fukuto, J.; Feelisch, M.; Wink, D.; Kass, D. Proc. Natl. Acad. Sci. U. S. A. 2001, 98, 10463.

[5] Switzer, C. H.; Flores-Santana, W.; Mancardi, D.; Donzelli, S.; Basudhar, D.; Ridnour, L. A.; Miranda, K. M.; Fukuto, J. M.; Paolocci, N.; Wink, D. A. Biochim. Biophys. Acta, Bioenerg. 2009, $1787,835$.

[6] Bult, H.; Boeckxstaens, G. E.; Pelckmans, P. A.; Jorfaens, F. H.; Van Maercke, Y. M.; Herman, A. G. Nature 1990, 345, 346.

[7] Irvine, J. C.; Kemp-Harper, B. K.; Widdop, R. E. Hypertension 2007, 49, 1468 .

[8] Lee, M. J. C.; Nagasawa, H. T.; Elberling, J. A.; Demaster, E. G. J. Med. Chem. 1992, 35, 3648 .
[9] Li, M.; Wang, Y.; Liu, G.; Lv, H.; Xing, G. Chin. J. Org. Chem. 2017, 37, 356 (in Chinese)

(李美含, 王宇童, 刘广建，吕海娟，邢国文，有机化学，2017, 37, 356.)

[10] Da Silva, T. R. M.; De Freitas, J. R.; Silva, Q. C.; Figueira, C. P.; Roxo, E.; Leao, S. C.; De Freitas, L. A. R.; Veras, P. S. T. Infect. Immun. 2002, 70, 5628.

[11] Radi, R. Proc. Natl. Acad. Sci. U. S. A. 2004, 101, 4003.

[12] Dong, B.; Kong, X.; Lin, W. ACS Chem. Biol. 2018, 13, 1714.

[13] Jing, X.; Yu, F.; Chen, L. Prog. Chem. 2014, 26, 866 (in Chinese). (景晓娅, 于法标, 陈令新, 化学进展, 2014, 26, 866.)

[14] Apfel, U.-P.; Buccella, D.; Wilson, J. J.; Lippard, S. J. Inorg. Chem. 2013, 52, 3285.

[15] Lv, H.; Ma, R.; Zhang, X.; Li, M.; Wang, Y.; Wang, S.; Xing, G Tetrahedron 2016, 72, 5495

[16] R., C. M.; P., T. J. J. Phys. Org. Chem. 2011, 24, 993

[17] Kawai, K.; Ieda, N.; Aizawa, K.; Suzuki, T.; Miyata, N.; Nakagawa, H. J. Am. Chem. Soc. 2013, 135, 12690.

[18] Liu, C.; Wu, H.; Wang, Z.; Shao, C.; Zhu, B.; Zhang, X. Chem. Commun. 2014, 50, 6013.

[19] Tan, Y.; Liu, R.; Zhang, H.; Peltier, R.; Lam, Y.; Zhu, Q.; Hu, Y.; Sun, H. Sci. Rep. 2015, 5. 16979.

[20] Gong, X.; Yang, X.; Zhong, Y.; Chen, Y.; Li, Z. Dyes Pigm. 2016, $131,24$.

[21] Jin, X.; Sun, X.; Di, X.; Zhang, X.; Huang, H.; Liu, J.; Ji, P.; Zhu, H. Sens. Actuators, B 2016, 224, 209.

[22] Ali, F.; Sreedharan, S.; Ashoka, A. H.; Saeed, H. K.; Smythe, C. G. W.; Thomas, J. A.; Das, A. Anal. Chem. 2017, 89, 12087.

[23] Zhou, Y.; Zhang, X.; Yang, S.; Li, Y.; Qing, Z.; Zheng, J.; Li, J.; Yang, R. Anal. Chem. 2017, 89, 4587.

[24] Li, H.; Yao, Q.; Xu, F.; Xu, N.; Ma, X.; Fan, J.; Long, S.; Du, J.; Wang, J.; Peng, X. Anal. Chem. 2018, 90, 4641.

[25] Yuan, S.; Wang, F.; Yang, G.; Lu, C.; Nie, J.; Chen, Z.; Ren, J.; Qiu, Y.; Sun, Q.; Zhao, C.; Zhu, W. H. Anal. Chem. 2018, 90, 3914.

[26] Jing, X.; Yu, F.; Chen, L. Chem. Commun. 2014, 50, 14253.

[27] Zhang, B. B.; Yang, X. P.; Zhang, R.; Liu, Y.; Ren, X. L.; Xian, M.; Ye, Y.; Zhao, Y. F. Anal. Chem. 2017, 89, 10384.

[28] Zheng, K.; Lin, W.; Cheng, D.; Chen, H.; Liu, Y.; Liu, K. Chem. Commun. 2015, 51, 5754.

[29] Zhu, X.; Xiong, M.; Liu, H.; Mao, G.; Zhou, L.; Zhang, J.; Hu, X.; Zhang, X.; Tan, W. Chem. Commun. 2016, 52, 733.

[30] Dong, B.; Song, X.; Kong, X.; Wang, C.; Zhang, N.; Lin, W. J. Mater. Chem. B 2017, 5, 5218.

[31] Fernández-Alonso, S.; Corrales, T.; Pablos, J. L.; Catalina, F. Sens. Actuators, B 2018, 270, 256.

[32] Tang, J.; Ma, S. G.; Zhang, D.; Liu, Y. Q.; Zhao, Y. F.; Ye, Y. Sens. Actuators, B 2016, 236, 109.

[33] Gupta, N.; Kaur, T.; Bhalla, V.; Parihar, R. D.; Ohri, P.; Kaur, G.; Kumar, M. Chem. Commun. 2017, 53, 12646.

[34] Zhang, B. B.; Qin, F. Y.; Niu, H. W.; Liu, Y.; Zhang, D.; Ye, Y. New J. Chem. 2017, 41, 14683.

[35] Xie, Z.; Fu, M.; Yin, B.; Zhu, Q. Chin. J. Org. Chem. 2018, 38, 1364 (in Chinese) (谢振达, 付曼琳, 尹彪, 朱勍, 有机化学, 2018, 38, 1364.)

[36] Wang, Y.; Liu, C.; Yang, W.; Zou, G.; Zhang, X.; Wu, F.; Yu, S.; Luo, X.; Zhou, X. Chem. Commun. 2018, 54, 1497.

[37] Liu, C.; Wang, Y.; Zhang, X.; Wu, F.; Yang, W.; Zou, G.; Yao, Q.; Wang, J.; Chen, Y.; Wang, S.; Zhou, X. Chem. Sci. 2017, 8, 4505.

[38] Christensen, K.; Myers, J.; Swanson, J. Cell Sci. 2002, 115, 599.

[39] Michael, S.; Raoul, K.; Michael, K.; Brian, H. Anal. Chem. 1996, 65,1414 .

(Li, L.; Fan, Y.) 